\title{
QUALITY OF LIFE AND POVERTY: MEASURING AND COMPARABILITY
}

\author{
MERCEDES MOLPECERES-ABELLA \\ Dept. de Economía Aplicada, Universidad de Valladolid, Avda. Valle de Esgueva 6, 47011 \\ Valladolid, Spain \\ abella@eco.uva.es \\ JOSÉ LUIS GARCÍA-LAPRESTA \\ PRESAD Research Group, BORDA Research Unit, IMUVA, Dept. de Economía Aplicada, \\ Universidad de Valladolid, Avda. Valle de Esgueva 6, 47011 Valladolid, Spain
}

lapresta@eco.uva.es

\begin{abstract}
In this paper, we analyze the correspondence among the rankings of the Spanish regions according to different measures of monetary poverty and quality of life, in 2012 . To do that, the Spearman's rank correlation coefficient is used. Different aggregation methods are applied to calculate the selected measures of poverty and quality of life. The monetary poverty measures aggregate the income gaps, while the quality of life measures aggregate a set of social indicators dealing with ten different domains. In both cases, among other traditional aggregation procedures, the exponential mean is used because its properties are especially adequate in these contexts.
\end{abstract}

Keywords: Quality of life; poverty; aggregation functions; exponential means; dual decomposition.

\section{Introduction}

For decades, poverty has been a central issue in Economy (see Rowntree ${ }^{31}$, Orshansky $^{29}$, Atkinson ${ }^{1,2}$ and Chakravarty and Muliere ${ }^{9}$, among others). Its definition and measurement can be based on the use of objective or subjective indicators and adopt an absolute or a relative approach $\left(\operatorname{see~Sen~}^{34,35}\right)$.

According to Eurostat ${ }^{15}$, the relative approach based on income has been chosen in Europe to show that poverty is related to social exclusion in accordance with many previous studies (see Townsend ${ }^{42}$ and Hagenaars ${ }^{22}$ ). So that, it can be assumed that being poor depends on the living conditions, not on how individuals feel about them (see Ringen ${ }^{30}$ ), and that poverty in advanced countries can be reasonably defined in relative terms as persons not having resources enough to achieve a minimum acceptable way of life in the country they belong to (see Townsend ${ }^{41}$ ). Then, population can be divided into the poor and the non-poor, according to an income poverty line or threshold set at a fixed percentage of the median income (see Fuchs ${ }^{17}$ ). This paper focuses on this kind of poverty measures and sets the poverty line at the $60 \%$ of the median equivalent disposable income, as in many national, 
regional and international studies (see Buhmann et $\mathrm{al}^{8}, \mathrm{OECD}^{26}$ and Atkinson ${ }^{2}$, among others). It allows us to identify the poor but the problem of aggregation still persists: how to combine income distribution and the poverty line in order to construct a poverty measure. Considering their properties (see Subramanian ${ }^{40}$ ), two different families of poverty measures have been selected to provide a comparative analysis for the Spanish regions (at NUTS2 level) in 2012: the Foster-GreerThorbecke class $^{16}$ and the one introduced by García-Lapresta et al. ${ }^{18}$. According to the Spearman's coefficient, it has been established that regional poverty rankings do not change significantly when the importance given to inequality among the poor is altered.

No doubt, poverty is important to rank societies from the best to the worst. But it has been suggested that a more varied set of characteristics should be taken into account to generate this kind of rankings. Literature on quality of life might be the answer to that requirement (see Stiglitz et al. ${ }^{39}$ ). Although it is a more elusive, controversial and complex than poverty, quality of life is usually defined by identifying its main domains (i.e., health status, social support, income, poverty, environmental quality, personal security, etc.). Among all the methodologies proposed to measure quality of life, the most widespread consists in aggregating a set of social indicators that capture its principal domains (see Nardo et al. ${ }^{25}$ and OECD ${ }^{27,28}$ ). Following these premises, we propose several tentative quality of life measures: one ordinal composite index (see Slottje ${ }^{36}$, Slottje et al. ${ }^{37}$ and Dasgupta and Weale ${ }^{11}$ ) as well as several cardinal composite indices based all of them in the same set of social indicators (see Booysen ${ }^{7}$ ). All of them are used to rank Spanish regions from the highest to the lowest quality of life. These new regional rankings do not change substantially according to Spearman's coefficient regardless of the composite quality of life measure used.

In fact, both types of regional rankings result to be surprisingly similar, although the information used to obtain them is obviously much more restrictive in the case of the monetary poverty rankings. None of these concordances have been obtained adopting a subjective approach to elaborate quality of life rankings based on satisfaction with life (see Diener ${ }^{13}$ ).

The rest of the paper is organized as follows. Section 2 is devoted to introduce basic notation and notions. In Section 3 we present the poverty measures used in this paper and we compare regional rankings on poverty obtained for Spanish regions in 2012. In Section 4 different quality of life measures are proposed. Spanish regions are then reordered according to them and differences among these new rankings are analyzed. Monetary poverty as a proxy for quality of life at regional level has also been evaluated. Finally, Section 5 contains some concluding remarks and further research. 


\section{Preliminaries}

Vectors in $[0, \infty)^{m}$ are denoted as $\boldsymbol{x}=\left(x_{1}, \ldots, x_{m}\right)$; in particular, $\mathbf{0}=(0, \ldots, 0)$ and $\mathbf{1}=(1, \ldots, 1)$. Given $\boldsymbol{x}, \boldsymbol{y} \in[0, \infty)^{m}$, by $\boldsymbol{x} \geq \boldsymbol{y}$ we mean $x_{i} \geq y_{i}$ for every $i \in\{1, \ldots, m\}$, and by $\boldsymbol{x}>\boldsymbol{y}$ we mean $\boldsymbol{x} \geq \boldsymbol{y}$ and $\boldsymbol{x} \neq \boldsymbol{y}$.

Given $\boldsymbol{x} \in[0, \infty)^{m}$, the increasing and decreasing reorderings of the coordinates of $\boldsymbol{x}$ are denoted as $x_{(1)} \leq \cdots \leq x_{(m)}$ and $x_{[1]} \geq \cdots \geq x_{[m]}$, respectively. Consequently, $x_{(1)}=\min \left\{x_{1}, \ldots, x_{m}\right\}=x_{[m]}, x_{(m)}=\max \left\{x_{1}, \ldots, x_{m}\right\}=x_{[1]}$ and $x_{[k]}=x_{(m-k+1)}$ for every $k \in\{1, \ldots, m\}$.

Given a set $I$, with $\# I$ we denote the cardinality of $I$.

A weak order (or complete preorder) is a complete and transitive binary relation. With $\succ$ and $\sim$ we denote the asymmetric and the symmetric parts of the weak order, respectively.

\subsection{Aggregation functions}

We now introduce standard properties of real functions on $[0,1]^{m}$ and aggregation functions. For more details, see Beliakov et al. ${ }^{6}$, Grabisch et al. ${ }^{21}$ and Beliakov et $a l .5$.

Definition 1. Let $A:[0,1]^{m} \longrightarrow \mathbb{R}$ be a function.

(1) $A$ is idempotent if for every $\boldsymbol{x} \in[0,1]: A(x \cdot \mathbf{1})=x$.

(2) $A$ is symmetric if for every permutation $\sigma$ on $\{1, \ldots, m\}$ and every $\boldsymbol{x} \in[0,1]^{m}$ : $A\left(x_{\sigma(1)}, \ldots, x_{\sigma(m)}\right)=A(\boldsymbol{x})$.

(3) $A$ is monotonic if for all $\boldsymbol{x}, \boldsymbol{y} \in[0,1]^{m}: \boldsymbol{x} \geq \boldsymbol{y} \Rightarrow A(\boldsymbol{x}) \geq A(\boldsymbol{y})$.

(4) $A$ is strictly monotonic if for all $\boldsymbol{x}, \boldsymbol{y} \in[0,1]^{m}: \boldsymbol{x}>\boldsymbol{y} \Rightarrow A(\boldsymbol{x})>A(\boldsymbol{y})$.

(5) $A$ is compensative if for every $\boldsymbol{x} \in[0,1]^{m}: x_{(1)} \leq A(\boldsymbol{x}) \leq x_{(m)}$.

(6) $A$ is self-dual if for every $\boldsymbol{x} \in[0,1]^{m}: A(\mathbf{1}-\boldsymbol{x})=1-A(\boldsymbol{x})$.

(7) $A$ is anti-self-dual if for every $\boldsymbol{x} \in[0,1]^{m}: A(\mathbf{1}-\boldsymbol{x})=A(\boldsymbol{x})$.

(8) $A$ is invariant for translations if for every $\boldsymbol{x} \in[0,1]^{m}$ and every $t \in \mathbb{R}$ such that $\boldsymbol{x}+t \cdot \mathbf{1} \in[0,1]^{m}: A(\boldsymbol{x}+t \cdot \mathbf{1})=A(\boldsymbol{x})$.

(9) $A$ is stable for translations if for every $\boldsymbol{x} \in[0,1]^{m}$ and every $t \in \mathbb{R}$ such that $\boldsymbol{x}+t \cdot \mathbf{1} \in[0,1]^{m}: A(\boldsymbol{x}+t \cdot \mathbf{1})=A(\boldsymbol{x})+t$.

Definition 2. Let $\left(A^{(m)}\right)_{m \in \mathbb{N}}$ be a sequence of functions, with $A^{(m)}:[0,1]^{m} \longrightarrow$ $\mathbb{R}$ and $A^{(1)}(x)=x$ for every $x \in[0,1]$.

(1) $\left(A^{(m)}\right)_{m \in \mathbb{N}}$ is invariant for replications if for all $\boldsymbol{x} \in[0,1]^{m}$ and any number of replications $t \in \mathbb{N}$ of $\boldsymbol{x}$ :

$$
A^{(t m)}(\overbrace{\boldsymbol{x}, \ldots, \boldsymbol{x}}^{t})=A^{(m)}(\boldsymbol{x}) .
$$

(2) $\left(A^{(m)}\right)_{m \in \mathbb{N}}$ is decomposable if, for any given subset of variables, every variable in the subset can have its value replaced by the partial aggregated value of the 
subset without altering the overall aggregated value of the full set of variables; for instance if

$$
\begin{aligned}
& A^{(m)}\left(x_{1}, \ldots, x_{k}, x_{k+1}, \ldots, x_{m}\right)= \\
& =A^{(m)}\left(A^{(k)}\left(x_{1}, \ldots, x_{k}\right), \ldots, A^{(k)}\left(x_{1}, \ldots, x_{k}\right), x_{k+1}, \ldots, x_{m}\right),
\end{aligned}
$$

for all $\boldsymbol{x} \in[0,1]^{m}$ and $k \in\{1, \ldots, m\}$.

Definition 3. A function $A:[0,1]^{m} \longrightarrow[0,1]$ is called an aggregation function if it is monotonic and satisfies the boundary conditions $A(\mathbf{0})=0$ and $A(\mathbf{1})=1$.

The dual decomposition of an aggregation function $A:[0,1]^{m} \longrightarrow[0,1]$ in the core

$$
\widehat{A}(\boldsymbol{x})=\frac{A(\boldsymbol{x})-A(\mathbf{1}-\boldsymbol{x})+1}{2}
$$

and the remainder

$$
\widetilde{A}(\boldsymbol{x})=A(\boldsymbol{x})-\widehat{A}(\boldsymbol{x})=\frac{A(\boldsymbol{x})+A(\mathbf{1}-\boldsymbol{x})-1}{2}
$$

can be found in García-Lapresta and Marques Pereira ${ }^{19}$.

Given $\alpha \neq 0$, the exponential mean $A_{\alpha}:[0,1]^{m} \longrightarrow[0,1]$ is the aggregation function defined as

$$
A_{\alpha}(\boldsymbol{x})=\frac{1}{\alpha} \ln \frac{e^{\alpha x_{1}}+\cdots+e^{\alpha x_{m}}}{m} .
$$

Every exponential mean is continuous, idempotent, symmetric, strictly monotonic, compensative, stable for translations and decomposable.

On the use of the dual decomposition of aggregation functions to welfare economics, see García-Lapresta et $a .^{18}$, Aristondo et $a l .^{3,4}$ and García-Lapresta and Marques Pereira ${ }^{20}$.

We now describe the dual decomposition of exponential means (see GarcíaLapresta and Marques Pereira ${ }^{19}$ for more details).

Given $\alpha \neq 0$, the core of $A_{\alpha}$ is the aggregation function $\widehat{A}_{\alpha}:[0,1]^{m} \longrightarrow[0,1]$ defined as

$$
\widehat{A}_{\alpha}(\boldsymbol{x})=\frac{1}{2 \alpha} \ln \frac{e^{\alpha x_{1}}+\cdots+e^{\alpha x_{m}}}{e^{-\alpha x_{1}}+\cdots+e^{-\alpha x_{m}}} .
$$

The core of every exponential mean is continuous, idempotent, symmetric, strictly monotonic, compensative, stable for translations, self-dual and invariant for replications. Consequently, it can be considered as a position measure.

Given $\alpha \neq 0$, the remainder of $A_{\alpha}$ is the mapping $\widetilde{A}_{\alpha}:[0,1]^{m} \longrightarrow \mathbb{R}$ defined as

$$
\widetilde{A}_{\alpha}(\boldsymbol{x})=\frac{1}{2 \alpha} \ln \frac{\left(e^{\alpha x_{1}}+\cdots+e^{\alpha x_{m}}\right)\left(e^{-\alpha x_{1}}+\cdots+e^{-\alpha x_{m}}\right)}{m^{2}} .
$$

The remainder of every exponential mean is continuous, symmetric, anti-selfdual, invariant for translations and invariant for replications. Additionally, $\widetilde{A}_{\alpha}(\boldsymbol{x})=$ 
0 if and only if $x_{1}=\cdots=x_{n}$. Consequently, it can be considered as an absolute dispersion measure in the sense of Martínez-Panero et al. ${ }^{24}$ whenever $\alpha>0$, since $\widetilde{A}_{\alpha}(\boldsymbol{x}) \geq 0$ for every $\boldsymbol{x} \in[0,1]^{m}$; if $\alpha<0$, then $\widetilde{A}_{\alpha}(\boldsymbol{x}) \leq 0$ for every $\boldsymbol{x} \in[0,1]^{m}$ and $\widetilde{A}_{\alpha}$ is an absolute dispersion measure excepting the sign, that now is negative.

We now show the parameter limits of the exponential means and their remainders (see Prop. 35 of García-Lapresta and Marques Pereira ${ }^{19}$ ):

(1) $\lim _{\alpha \rightarrow \infty} A_{\alpha}(\boldsymbol{x})=x_{(n)}$

(2) $\lim _{\alpha \rightarrow-\infty} A_{\alpha}(\boldsymbol{x})=x_{(1)}$.

(3) $\lim _{\alpha \rightarrow \infty} \widetilde{A}_{\alpha}(\boldsymbol{x})=\frac{x_{(n)}-x_{(1)}}{2}$.

(4) $\lim _{\alpha \rightarrow-\infty} \widetilde{A}_{\alpha}(\boldsymbol{x})=-\frac{x_{(n)}-x_{(1)}}{2}$

It is important noticing that $A_{\alpha}(\boldsymbol{x})=\widehat{A}_{\alpha}(\boldsymbol{x})+\widetilde{A}_{\alpha}(\boldsymbol{x})$, for every $\boldsymbol{x} \in[0,1]^{m}$.

\subsection{Spearman's rank correlation coefficient}

Let $S$ be a weak order on the set of objects $O=\left\{o_{1}, \ldots, o_{m}\right\}$. The position of object $o_{i} \in O$ in $S$ is defined as

$$
p_{S}\left(o_{i}\right)=m-\#\left\{o_{j} \in X \mid o_{i} \succ o_{j}\right\}-\frac{1}{2} \#\left\{o_{j} \in\left(O \backslash\left\{o_{i}\right\}\right) \mid o_{j} \sim o_{i}\right\} .
$$

These positions can be also obtained as the average of the corresponding ones after a linearization process (see Smith ${ }^{38}$ and Cook and Seiford ${ }^{10}$ ).

Example 1. Consider the weak order $S$ on $O=\left\{o_{1}, \ldots, o_{8}\right\}$ depicted as follows

$$
\begin{gathered}
S \\
\hline O_{2} O_{5} \\
O_{1} \\
O_{3} O_{7} O_{8} \\
O_{4} O_{6}
\end{gathered}
$$

Then,

$$
\begin{aligned}
& p_{S}\left(o_{2}\right)=p_{S}\left(o_{5}\right)=8-6-\frac{1}{2} 1=1.5=\frac{1+2}{2} \\
& p_{S}\left(o_{1}\right)=8-5-\frac{1}{2} 0=3 \\
& p_{S}\left(o_{3}\right)=p_{S}\left(o_{7}\right)=p_{S}\left(o_{8}\right)=8-2-\frac{1}{2} 2=5=\frac{4+5+6}{3} \\
& p_{S}\left(o_{4}\right)=p_{S}\left(o_{6}\right)=8-0-\frac{1}{2} 1=7.5=\frac{7+8}{2}
\end{aligned}
$$


Definition 4. Given two weak orders $S$ and $T$ on $O=\left\{o_{1}, \ldots, o_{m}\right\}$, the Spearman's rank correlation coefficient between $S$ and $T$ is defined as

$$
\rho(S, T)=\frac{\frac{1}{m} \cdot \sum_{i=1}^{m}\left(\left(p_{S}\left(o_{i}\right)-\frac{1}{m} \sum_{i=1}^{m} p_{S}\left(o_{i}\right)\right) \cdot\left(p_{T}\left(o_{i}\right)-\frac{1}{m} \sum_{i=1}^{m} p_{T}\left(o_{i}\right)\right)\right)}{\sqrt{\frac{1}{m} \cdot \sum_{i=1}^{m}\left(p_{S}\left(o_{i}\right)-\frac{1}{m} \sum_{i=1}^{m} p_{S}\left(o_{i}\right)\right)^{2} \cdot \frac{1}{m} \cdot \sum_{i=1}^{m}\left(p_{T}\left(o_{i}\right)-\frac{1}{m} \cdot \sum_{i=1}^{m} p_{T}\left(o_{i}\right)\right)^{2}}} .
$$

Taking into account that

$$
\sum_{i=1}^{m} p_{S}\left(o_{i}\right)=\sum_{i=1}^{m} p_{T}\left(o_{i}\right)=\frac{(m+1) \cdot m}{2}
$$

and after some simplifications, we obtain

$$
\rho(S, T)=\frac{\sum_{i=1}^{m}\left(\left(p_{S}\left(o_{i}\right)-\frac{m+1}{2}\right) \cdot\left(p_{T}\left(o_{i}\right)-\frac{m+1}{2}\right)\right)}{\sqrt{\sum_{i=1}^{m}\left(p_{S}\left(o_{i}\right)-\frac{m+1}{2}\right)^{2} \cdot \sum_{i=1}^{m}\left(p_{T}\left(o_{i}\right)-\frac{m+1}{2}\right)^{2}}} .
$$

This coefficient lies between -1 and 1 . When two weak orders are identical (perfect positive correlation) it follows that $\rho(S, T)=1$. If one is the reverse of the other (perfect negative correlation), then $\rho(S, T)=-1$. The higher is the absolute value of the coefficient, the stronger is the intensity of rank correlation.

The statistic for testing the null hypothesis of independence $H_{0}, \rho(S, T)=0$, is:

$$
t_{m-2}=\frac{\rho(S, T) \sqrt{m-2}}{\sqrt{1-(\rho(S, T))^{2}}}
$$

according to a Student's $t$-distribution with $m-2$ degrees of freedom.

\section{Poverty}

We consider a population consisting of $n$ individuals, with $n \geq 2$. An income distribution is represented by a vector $\boldsymbol{x}=\left(x_{1}, \ldots, x_{n}\right) \in[0, \infty)^{n}$, where $x_{i}$ represents the income of individual $i \in\{1, \ldots, n\}$.

According to $\mathrm{Sen}^{33}$, a poverty measure consists essentially of an aggregation procedure within the poor sector of the population. The identification of the poor individuals requires the specification of a poverty line $z \in(0, \infty)$ which represents the necessary income to maintain a minimum level of living. Given an income distribution $\boldsymbol{x}$ an individual is considered to be poor if his/her income is below the poverty line. Otherwise the individual is non-poor. 
The set of poor individuals in the population is denoted by

$$
Q(\boldsymbol{x}, z)=\left\{i \in\{1, \ldots, n\} \mid x_{i}<z\right\},
$$

and $q(\boldsymbol{x}, z)$ denotes the number of the poor, $q(\boldsymbol{x}, z)=\# Q(\boldsymbol{x}, z)$.

Definition 5. For all $\boldsymbol{x} \in[0, \infty)^{n}$ and $z \in(0, \infty)$, the normalized gap of individual $i$ is defined as

$$
g_{i}=\max \left\{\frac{z-x_{i}}{z}, 0\right\}
$$

Notice that $g_{i} \in[0,1], g_{i}=0 \Leftrightarrow x_{i} \geq z$, and $g_{i}=1 \Leftrightarrow x_{i}=0$. In addition, the normalized gaps are invariant under proportional income changes, i.e., the function $G:[0, \infty)^{n} \times(0, \infty) \longrightarrow[0,1]^{n}$ defined as $G(\boldsymbol{x}, z)=\left(g_{1}, \ldots, g_{n}\right)$ is homogeneous of degree $0: G\left(\lambda \cdot x_{1}, \ldots, \lambda \cdot x_{n}, \lambda \cdot z\right)=G\left(x_{1}, \ldots, x_{n}, z\right)$ for every $\lambda>0$.

We now introduce a special notation for the incomes and normalized gaps of the poor individuals in the population: $q=q(\boldsymbol{x}, z), \boldsymbol{x}_{p}=\left(x_{(1)}, \ldots, x_{(q)}\right)$ with $x_{(1)} \leq \cdots \leq x_{(q)}<z$, and $\boldsymbol{g}_{p}=\left(g_{[1]}, \ldots, g_{[q]}\right)$ with $g_{[1]} \geq \cdots \geq g_{[q]}>0$, and $g_{[i]}=\left(z-x_{(i)}\right) / z$ for $i=1, \ldots, q$.

\subsection{Poverty measures}

There exist in the literature a number of poverty measures. According to $\mathrm{Sen}^{33}$ and Jenkins and Lambert ${ }^{23}$, every poverty measure should be expressed as a function of three poverty indicators, the so called three I's: incidence, intensity and inequality of poverty.

For our analysis we have selected two families of parameterized poverty measures that meet at least two out of the three Sen's requirements: the poverty measures proposed by Foster et al. ${ }^{16}$ and García-Lapresta et al. ${ }^{18}$.

First we introduce the Foster-Greer-Thorbecke (FGT) poverty measures.

Definition 6. Given $\alpha \in[0, \infty)$, the $F G T_{\alpha}$ poverty measure is the function $F G T_{\alpha}:[0, \infty)^{n} \times(0, \infty) \longrightarrow[0,1]$ defined as

$$
F G T_{\alpha}(\boldsymbol{x}, z)=\frac{1}{n} \sum_{i=1}^{q} g_{[i]}^{\alpha} .
$$

Notice that $F G T_{0}$ is the headcount ratio,

$$
H(\boldsymbol{x}, z)=\frac{q}{n},
$$

the first measure introduced in the literature. It is rejected because it measures the incidence of poverty, but it does not measure the intensity or the inequality of poverty. In turn, $F G T_{1}$ is the poverty gap ratio, that measures incidence as well as intensity of poverty. For higher values of the parameter $\alpha$, the three I's are considered. 
The next poverty measures were introduced and analyzed by García-Lapresta et al. ${ }^{18}$.

Definition 7. Given $\alpha \in(0, \infty)$, the poverty measure associated with $A_{\alpha}$ is the function $P_{\alpha}:[0, \infty)^{n} \times(0, \infty) \longrightarrow[0,1]$ defined as

$$
P_{\alpha}(\boldsymbol{x}, z)= \begin{cases}H(\boldsymbol{x}, z) \cdot A_{\alpha}\left(\boldsymbol{g}_{p}\right)=\frac{q}{n} \cdot \frac{1}{\alpha} \ln \frac{e^{\alpha g_{[1]}}+\cdots+e^{\alpha g_{[q]}}}{q}, & \text { if } q \neq 0 \\ 0, & \text { if } q=0\end{cases}
$$

The poverty measure $P_{\alpha}$ satisfies some interesting properties (see GarcíaLapresta et al. ${ }^{18}$ ):

(1) Poverty Focus: poverty should not depend on the non-poor incomes.

(2) Normalization: if all the individuals are non-poor, then the society deprivation level is equal to 0 .

(3) Poverty Symmetry: no other characteristic apart from the income deprivation matters in defining a poverty index.

(4) Replication Invariance: if the population is replicated, then poverty should not change; this allows comparing populations of different sizes.

(5) Poverty Monotonicity: poverty should increase if a poor income decreases.

(6) Transfer Sensitivity: greater weight should be placed on the poorer incomes and poverty should decrease if inequality among the poor decreases.

(7) Diminishing Transfer Sensitivity: the poverty reduction effect of a "poor to poorer" progressive transfer should decrease as the income of the poorer person increases.

The axiomatic approach, together with the presence of the three I's of poverty, set a list of properties that any reasonable poverty measure should satisfy. A number of different axioms exist in literature and the consistency among them have been widely analyzed (see, for instance, Donaldson and Weymark ${ }^{14}$ ). Table 1 summarizes the properties satisfied for each of the considered poverty measures.

Table 1. Properties of the poverty measures.

\begin{tabular}{lcccc}
\hline Property & $F G T_{0}$ & $F G T_{1}$ & $F G T_{\alpha}(\alpha>1)$ & $P_{\alpha}$ \\
\hline Poverty Focus & Yes & Yes & Yes & Yes \\
Normalization & Yes & Yes & Yes & Yes \\
Poverty Symmetry & Yes & Yes & Yes & Yes \\
Replication Invariance & Yes & Yes & Yes & Yes \\
Poverty Monotonicity & No & Yes & Yes & Yes \\
Transfer Sensitivity & No & No & Yes & Yes \\
Diminishing Transfer Sensitivity & No & No & Yes & Yes \\
\hline
\end{tabular}


Table 2 shows whether the three I's (incidence, intensity and inequality) are captured by each of the considered poverty measures.

Table 2. The three I's of the poverty measures.

\begin{tabular}{lcccc}
\hline Property & $F G T_{0}$ & $F G T_{1}$ & $F G T_{2}$ & $P_{\alpha}$ \\
\hline Incidence & Yes & Yes & Yes & Yes \\
Intensity & No & Yes & Yes & Yes \\
Inequality & No & No & Yes & Yes \\
\hline
\end{tabular}

According to García-Lapresta et al. ${ }^{18}$, for every $\alpha>0$, the poverty measure $P_{\alpha}$ associated with $A_{\alpha}$ can be decomposed in the following way:

$$
P_{\alpha}(\boldsymbol{x}, z)= \begin{cases}H(\boldsymbol{x}, z) \cdot\left(\widehat{A}_{\alpha}\left(\boldsymbol{g}_{p}\right)+\widetilde{A}_{\alpha}\left(\boldsymbol{g}_{p}\right)\right), & \text { if } q \neq 0, \\ 0, & \text { if } q=0 .\end{cases}
$$

Thus, the poverty measure $P_{\alpha}$ is clearly expressed through the three I's (incidence, intensity and inequality), by means of $H, \widehat{A}_{\alpha}$ and $\widetilde{A}_{\alpha}$, respectively. $H$ is the classical measure for estimating the poverty incidence. As mentioned above, $\widehat{A}_{\alpha}$ is continuous, idempotent, symmetric, strictly monotonic, compensative, stable for translations, self-dual and invariant for replications, therefore it can be considered as a good measure of the poverty intensity when it is applied to the normalized gaps of poor individuals. In turn, $\widetilde{A}_{\alpha}$ is continuous, symmetric, anti-self-dual, invariant for translations, invariant for replications, and it is 0 if and only if all the inputs are the same. Consequently, $\widetilde{A}_{\alpha}$ is a good measure of the poverty inequality when it is applied to the normalized gaps of poor individuals.

\subsection{Regional poverty comparisons in Spain}

As a case of study, we propose testing at ordinal level the consistency of the poverty analysis for the Spanish regions at NUT2 level, following the Eurostat's nomenclature (see Figure 1 and Table 3), using simultaneously the two families of parameterized poverty measures presented in Subsection 3.1: $F G T_{\alpha}(\alpha \geq 1)$ and $P_{\alpha}$.

Spanish regions have been ranked, the region with the lowest level of poverty achieving the first position, and so on, according to six poverty measures corresponding to different values of $\alpha$ for the two families of poverty measures defined above. All of them present different levels of sensibility towards people living with lower levels of income. In particular, the higher the parameter $\alpha$, the bigger the level of sensitivity of each measure towards the poorest population, and the higher the poverty measure value obtained.

Estimates for the disposable household income have been taken from the European Living Conditions Survey (available in Eurostat database). Assuming that 
Table 3. Spanish regions and their codes.

\begin{tabular}{ll}
\hline Code & NUTS 2 \\
\hline ES11 & Galicia \\
ES12 & Principado de Asturias \\
ES13 & Cantabria \\
ES21 & País Vasco \\
ES22 & Comunidad Foral de Navarra \\
ES23 & La Rioja \\
ES24 & Aragón \\
ES30 & Comunidad de Madrid \\
ES41 & Castilla y León \\
ES42 & Castilla - La Mancha \\
ES43 & Extremadura \\
ES51 & Catalunya \\
ES52 & Comunidad Valenciana \\
ES53 & Illes Balears \\
ES61 & Andalucía \\
ES62 & Región de Murcia \\
ES70 & Canarias \\
\hline
\end{tabular}

households enjoy economies of scale in consumption, an equivalence scale is needed to approximate an equivalent concept of income able to reflect differences in wellbeing derived from differences in household sizes. As there is not an accepted method for determining equivalence scales, here we use the parametric family of equivalence scales proposed by Buhmann et al. ${ }^{8}$.

Definition 8. Let $X_{h}$ be the disposable income for household $h$ and $n_{h}$ its size (the number of persons living together in the household pooling incomes and sharing consumption options). The equivalent income, $x_{i}$, is defined as

$$
x_{i}=\frac{X_{h}}{n_{h}^{\theta}},
$$

where $\theta \in[0,1]$ represents the economies of scale derived from household consumption.

The larger the parameter $\theta$, the smaller the economies of scale assumed.

In our calculations we have applied moderate economies of scale, $\theta=0.5$. Equivalent income for every household is assigned to all its members, assuming that equivalent disposable income is pooled and shared equally among all of them.

Equivalent disposable incomes are then used to calculate all the poverty measures selected to rank Spanish regions and test the consistency between them using the Spearman's rank correlation coefficient.

Do regional poverty rankings change substantially as the parameter $\alpha$ is modified? Table 4 includes estimated Spearman's rank correlation coefficients for six 


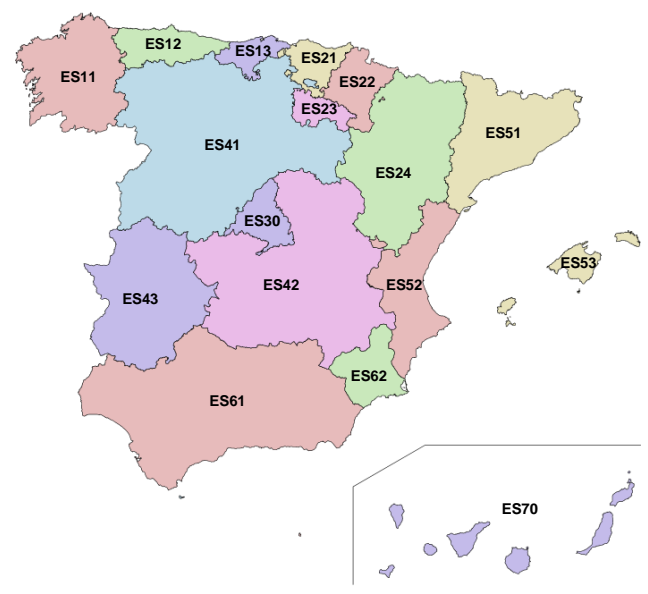

Fig. 1. Spain map regions.

different poverty measures.

Table 4. Spearman's rank correlation coefficients.

\begin{tabular}{lcccccc}
\hline & $F G T_{1}$ & $F G T_{2}$ & $F G T_{10}$ & $P_{1}$ & $P_{2}$ & $P_{10}$ \\
\hline$F G T_{1}$ & 1 & 0.9461 & 0.5907 & 0.9975 & 0.9951 & 0.9387 \\
$F G T_{2}$ & & 1 & 0.6985 & 0.9412 & 0.9387 & 0.8529 \\
$F G T_{10}$ & & & 1 & 0.5833 & 0.6005 & 0.6618 \\
$P_{1}$ & & & & 1 & 0.9975 & 0.9412 \\
$P_{2}$ & & & & & 1 & 0.9485 \\
$P_{10}$ & & & & & & 1 \\
\hline
\end{tabular}

All the estimated values for the correlation coefficients are statistically significant (at the 0.05 level of significance). Estimated coefficients are near the unit, except for those corresponding to $F G T_{10}$ that do not exceed 0.7. Probably because of its much higher sensibility towards the poorest population. Except for $F G T_{10}$, two regions out of the seventeen considered maintain the same position whatever the measure of poverty used (ES61 and ES70), both at the bottom of the ranking. Five regions lose only one position (ES21, ES22, ES24, ES51 and ES52), two of them being at 
the top of the ranking. And for other four regions (ES13, ES30, ES42 and ES53) the differences among their ranks do not exceed two positions (see Table 5). Therefore, results suggest a high level of consistency among relative regional positions based on income poverty, regardless of the measure or the level of sensibility towards poor people with lower income levels.

Table 5. Ranks of poverty in the Spanish regions.

\begin{tabular}{lcccccc}
\hline Region & $F G T_{1}$ & $F G T_{2}$ & $F G T_{10}$ & $P_{1}$ & $P_{2}$ & $P_{10}$ \\
\hline ES11 Galicia & 3 & 3 & 9 & 3 & 4 & 6 \\
ES12 Principado de Asturias & 9 & 9 & 4 & 9 & 9 & 5 \\
ES13 Cantabria & 6 & 8 & 12 & 6 & 6 & 7 \\
ES21 País Vasco & 2 & 2 & 1 & 2 & 2 & 1 \\
ES22 Comunidad Foral de Navarra & 1 & 1 & 6 & 1 & 1 & 2 \\
ES23 La Rioja & 12 & 16 & 17 & 12 & 12 & 10 \\
ES24 Aragón & 8 & 7 & 11 & 7 & 7 & 8 \\
ES30 Comunidad de Madrid & 5 & 6 & 3 & 5 & 5 & 4 \\
ES41 Castilla y León & 7 & 5 & 8 & 8 & 8 & 9 \\
ES42 Castilla - La Mancha & 16 & 14 & 5 & 16 & 16 & 14 \\
ES43 Extremadura & 14 & 11 & 10 & 14 & 14 & 16 \\
ES51 Catalunya & 4 & 4 & 2 & 4 & 3 & 3 \\
ES52 Comunidad Valenciana & 13 & 12 & 14 & 13 & 13 & 13 \\
ES53 Illes Balears & 11 & 13 & 15 & 11 & 11 & 11 \\
ES61 Andalucía & 15 & 15 & 13 & 15 & 15 & 15 \\
ES62 Región de Murcia & 10 & 10 & 7 & 10 & 10 & 12 \\
ES70 Canarias & 17 & 17 & 16 & 17 & 17 & 17 \\
\hline
\end{tabular}

It is generally accepted that societies with low levels of poverty, however it was defined and measured, are preferred to those with high levels of poverty contributing to raising quality of life. Considering the consistency shown by the regional rankings based on different measures of poverty, the question arises about what would be the effect on rankings if other social indicators related to quality of life were included. The approach of the synthetic measures of the quality of life can help us to answer that question, since its sole purpose is to summarize the most relevant information provided by a set of the quality of life indicators.

Next section reviews the guidelines of the huge literature devoted to construct synthetic measures. An aggregation function consistent with the exponential means, $A_{\alpha}$, as it is the family of poverty measures $P_{\alpha}$ used above, is then selected. The resulting synthetic measure of quality of life is used to rank regions. Finally, regional rankings on poverty and quality of life are compared by using the Spearman's coefficient. 


\section{Quality of life}

It may not be possible to define an objective synthetic measure of quality of life, but societies have to evaluate alternative social states. Organized objective data is needed to closely monitor social well-being. Research has resulted in different quality of life composite indices (for pros and cons of composite issues, see Nardo et al. $\left.{ }^{25}\right)$.

According to the principle of parsimony, the proposed Composite Measures of Quality of life (CMQ) are based on a selection of few relevant social indicators dealing with different issues (like health or inequality) that affect individuals and society as a whole. Controversial indicators have been excluded. Indicators interacting with each other, yet not measuring the same phenomena, have been included (for example, income and inequality).

Many different methodologies can be used to evaluate social quality of life and results depend on the methodology finally applied. None of them have been generally accepted for any society at any time, but experts agree that a high degree of transparency is required about methodological choices. Among all of them, composite indices provide clear advantages over competing methodologies: they have a multidimensional nature but, at the same time, synthesize numerous indicators into a single number which facilitates comparisons among territories and over time. Composite indexing entails the aggregation of different social indicators. It involves three steps: data selection (dimensions and indicators), standardization and aggregation.

\subsection{Data selection}

Measuring quality of life requires selecting social indicators that suitably capture any of its dimensions. They must be chosen on the basis of their analytical soundness, measurability, region coverage and relevance to the quality of life. There is no single definitive set of dimensions or indicators and proxy measures have to be used when desired data is unavailable. Each indicator, viewed individually, will show a social problem improving or worsening. All of them interrelate and contribute to the overall quality of life. We consider seven dimensions and ten social indicators (see Table 6 for more details).

- Dimension 1: Material living conditions.

1. Average equivalized disposable income: The equivalized income attributed to each member of the household is calculated by dividing the total disposable income of the household by the square root of the household size.

2. Severe material deprivation: Share of individuals having living conditions greatly constrained by a lack of resources and unable to afford at least four of the following: to pay rent or utility bills; to keep their home adequately warm; to pay unexpected expenses; to eat meat, fish or a protein equivalent every second day; a week holiday away from home; a car; a washing 
machine a colour TV; a telephone.

- Dimension 2: Inequality.

3. Income inequality: Gini coefficient for equivalized disposable income.

- Dimension 3: Health status.

4. Life expectancy: Life expectancy at birth.

5. Population without disabilities: Share of individuals aged over 15 without problems of mobility, for self-care or daily activities or suffering pain, discomfort, anxiety or depression.

- Dimension 4: Work and human capital.

6. Unemployment: Unemployment rate.

7. Human capital: Share of tertiary education employed in the science and technology sector.

- Dimension 5: Social capital.

8. Help from others: Share of individuals who have the possibility to ask for help (any kind of help: moral, material or financial) from any relatives, friends or neighbours who don't live in his or her household.

- Dimension 6: Personal security.

9. Crime: Share of individuals who have crime, violence or vandalism problems related to the place where they live.

- Dimension 7: Environmental quality.

10. Environmental problems: Share of individuals who have pollution, grime or other environmental problems related to the place where they live such as: smoke, dust, unpleasant smells or polluted water.

Table 6. Selected social indicators.

\begin{tabular}{llll}
\hline Indicator & Source & Year & Quality of life \\
\hline & & & \\
1. Average equivalized disposable income & EU-SILC & 2012 & increases \\
2. Severe material deprivation & EU-SILC & 2012 & decreases \\
3. Income inequality & Own* & 2012 & decreases \\
4. Life expectancy & Eurostat & 2012 & increases \\
5.Population without disabilities & INE & 2012 & increases \\
6. Unemployment & INE & 2012 & decreases \\
7. Human capital & INE & 2012 & increases \\
8. Help from others & EU-SILC & 2012 & increases \\
9. Crime & EU-SILC & 2012 & decreases \\
10. Environmental problems & EU-SILC & 2012 & decreases \\
\hline
\end{tabular}

* Own calculation based on EU-SILC 


\subsection{Standardization}

The essential reason why it may be necessary to scale variables is that raw data can have significantly different ranges. So that, without scaling, composite indices will be implicitly weighted towards variables with large ranges. This will imply that small but meaningful changes in an indicator will insignificantly affect the composite index.

The Linear Scaling Technique (LST) is likely to be the most common procedure to standardize the ranges of variables, including the Human Development Index ${ }^{43}$. It deals with the directionality issue and provides a consistent way to aggregate variables when some of them contribute to increase and others to decrease quality of life ${ }^{32}$. LST assumes that the empirically observed values of a variable represent its feasible range and that movement in it can be best expressed as a fraction of that feasible range. To do this, an estimate is made for the highest (max) and lowest (min) values for all regions.

Whenever there is a variable increase which corresponds to an increase in the overall quality of life, then it is scaled according to the following formula

$$
I_{k}^{j}=\frac{X_{k}^{j}-\min X_{k}}{\max X_{k}-\min X_{k}},
$$

where $I_{k}^{j}$ is the index score corresponding to the $k$-th social indicator for the region $j$ and $\max X_{k}$ and $\min X_{k}$ are the maximum and minimum values of the $k$-th social indicator for all the considered regions, respectively.

In contrast, whenever a variable increase corresponds to a decrease in the overall quality of life, then it is scaled according to the following formula

$$
I_{k}^{j}=\frac{\max X_{k}-X_{k}^{j}}{\max X_{k}-\min X_{k}} .
$$

In both cases, the ranges of values are in the unit interval, where the lowest level of quality of life is scored at 0 and the highest is set at 1 .

\subsection{Aggregation}

Probably, aggregation is the most controversial issue in the construction of composite indices. A standard approach to aggregation is the addition of all components to form the composite index. We have selected two different aggregation methods. An ordinal one, based on the Borda rule. And, accordingly with one of the poverty measures used above, $P_{\alpha}$, the exponential means. Both of them are advantageous because of its methodological transparency. In addition, exponential means can be decomposed into two components: the core; and the remainder, which measures to what extent disparities among different social indicators contribute to reduce quality of life. It shows preference for regions with similar values for all the social indicators considered.

As discussed in Booysen ${ }^{7}$, the most widely accepted and used techniques to set different weights in aggregations are three. First, develop specific surveys or pools 
on the relative importance of each of the facets of the quality of life considered. Second, ask experts or policy makers for their relative valuations. Both assignments of weights suffer from disadvantages linked to the fact that people's preferences can be non-transitive, specially when the number of variables grows. The third alternative is the use of statistical techniques such as Principal Component Analysis, which usually result on small weights for social indicators with little variation despite of their intrinsic importance for quality of life.

For now, equal weights are given to all the standardized variables, as in Slottje ${ }^{36}$ and in the Human Development Index ${ }^{43}$.

The ordinal Composite Measure of Quality of life for the region $j, C M Q_{o r}$, corresponds just to the sum of a region positions in the rankings according to all the standardized social indicators considered:

$$
C M Q_{\text {or }}=\sum_{k=1}^{10} p_{k}^{j}
$$

where $p_{k}^{j}$ is the position of region $j$ in the weak order dealing with index $k$.

In turn, the family of cardinal Composite Measures of Quality of life $C M Q_{\alpha}^{j}$, with $\alpha<0$, correspond to the exponential mean of the scores $I_{k}^{j}$ for each region $j$ :

$$
C M Q_{\alpha}^{j}=A_{\alpha}\left(I_{1}^{j}, \ldots, I_{10}^{j}\right)=\frac{1}{\alpha} \ln \sum_{k=1}^{10} \frac{e^{\alpha I_{k}^{j}}}{10} .
$$

We have considered $\alpha<0$ for ensuring that $C M Q_{\alpha}^{j}$ will be smaller in regions with marked deficiencies in any of the considered aspects of quality of life, even though they were well placed with respect to the rest of them. That is, in regions with similar values for all the indices $I_{k}^{j}$.

All the synthetic indices proposed above have been calculated for the Spanish regions in 2012. Estimations have been used to rank regions according to quality of life. According to each synthetic index, first position has been assigned to the region with the highest value, that is, the highest quality of life, and so on. This results in so many regional rankings as quality of life indices obtained. The issue about their consistency arises again, and Spearman's rank correlation coefficient is used to analyse this matter (see Table 7).

Table 7. Spearman's rank correlation coefficients. Quality of life indices.

\begin{tabular}{lcccc}
\hline & $C M Q_{\text {or }}$ & $C M Q_{-1}$ & $C M Q_{-2}$ & $C M Q_{-10}$ \\
\hline$C M Q_{o r}$ & 1 & 0.9975 & 0.9902 & 0.9044 \\
$C M Q_{-1}$ & & 1 & 0.9951 & 0.9191 \\
$C M Q_{-2}$ & & & 1 & 0.9338 \\
$C M Q_{-10}$ & & & & 1
\end{tabular}


Our results suggest that all the rankings, based on ordinal aggregation as well as cardinal aggregation, even for very high absolute values of $\alpha$, tend to assign very similar positions to the Spanish regions in 2012 according to quality of life. That is, the Spearman's coefficients are significant (at the 0.05 level of significance) and very close to 1 .

In fact, five regions out of the seventeen considered maintain the same position whatever the measure of quality of life used (ES21, ES22, ES24, ES61 and ES70), three of them are at the bottom of the ranking. Four regions lose only one position (ES13, ES30, ES51 and ES53), two of them being at the end of the ranking. And other five regions (ES11, ES22, ES41, ES42 and ES62) present differences between ranks that do not exceed two positions (see Table 8). Again, as in the poverty case, the consistency among different measures is very high for the Spanish regions in 2012 .

Table 8. Quality of life in the Spanish regions.

\begin{tabular}{lcccc}
\hline Region & $C M Q_{o r}$ & $C M Q_{-1}$ & $C M Q_{-2}$ & $C M Q_{-10}$ \\
\hline ES11 Galicia & 9 & 9 & 9 & 7 \\
ES12 Principado de Asturias & 8 & 8 & 8 & 11 \\
ES13 Cantabria & 5 & 5 & 6 & 6 \\
ES21 País Vasco & 4 & 4 & 4 & 4 \\
ES22 Comunidad Foral de Navarra & 1 & 1 & 1 & 1 \\
ES23 La Rioja & 6 & 6 & 5 & 3 \\
ES24 Aragón & 2 & 2 & 2 & 2 \\
ES30 Comunidad de Madrid & 7 & 7 & 7 & 8 \\
ES41 Castilla y León & 3 & 3 & 3 & 5 \\
ES42 Castilla - La Mancha & 11 & 10 & 10 & 9 \\
ES43 Extremadura & 10 & 11 & 12 & 15 \\
ES51 Catalunya & 13 & 13 & 13 & 14 \\
ES52 Comunidad Valenciana & 14 & 14 & 14 & 10 \\
ES53 Illes Balears & 12 & 12 & 11 & 12 \\
ES61 Andalucía & 16 & 16 & 16 & 16 \\
ES62 Región de Murcia & 15 & 15 & 15 & 13 \\
ES70 Canarias & 17 & 17 & 17 & 17 \\
\hline
\end{tabular}

As mentioned above, the $C M Q_{\alpha}^{j}$, and in fact any exponential mean, can be decomposed as the sum of two components. One of them, called remainder, is associated with the differences among estimated values for each region's quality of life indices. The smaller the differences among one region's indices, the smaller the remainder value. And, therefore, a low remainder value shows more similar quality of life situations attending to all the considered aspects. ES22 (Comunidad Foral de Navarra), the region with the highest quality of life, looking at any of the measures used, presents the lowest discount in quality of life associated to differences among 
indices, that is: its top position in the ranking is associated to high values in all the indicators selected. ES70 (Canarias) is in the opposite situation, at the bottom of the ranking and with a low discount because of the remainder component, that is: its bad position in the ranking is due to low quality of life indices in all the considered aspects.

Regions with the highest discounts linked to differences among indices are ES43 (Extremadura), at the bottom of the ranking, and ES30 (Comunidad de Madrid) and ES12 (Principado de Asturias), around the middle of it. For example, Comunidad de Madrid presents the highest levels for life expectancy, share of population without disabilities and tertiary educated employed in science and technology sector, but at the same time very high shares of individuals who declare suffering from crime and environmental problems. Data suggests that in Spanish regions in 2012 high or low differences among indices can be found at any position in the quality of life ranking.

\subsection{Comparing poverty and quality of life rankings for the Spanish regions}

The main objective is now analyze if poverty measures used in Section 3, so consistent among them, are good proxies for the composite measures of quality of life proposed in this section, which are also consistent among them. In other words, if regional rankings on poverty and those on quality of life are equivalent or not for Spanish regions in 2012. Spearman's rank correlation coefficients are still high (see Table 9), although smaller that those obtained above (see Table 4 and Table 7). No doubt it is due to the different nature of the concepts compared, being quality of life much broader than income poverty. In any case, it is noteworthy that most of the Spearman coefficients, close to 0.6, and statistically significant. Except those corresponding to $F G T_{10}$, probably because of the higher importance given to big poverty income gaps. Concluding, data suggests that the Spanish regions where income poverty is low (on the top of the poverty ranking) tend to be regions where quality of life is high (top positions on the quality of life ranking). This makes poverty measures acceptable proxies for the Spanish regions' quality of life in 2012

\subsection{Subjective quality of life}

On the basis that what constitutes a good life can be defined as the kind of life that is desirable for people, and assuming that individuals know more about their own life situation than anyone else, surveys on Life Satisfaction (LS) can be a valuable source of information to study quality of life. These surveys inform about how a respondent evaluates his or her life taken as a whole from 0 (not satisfied at all) to 10 (completely satisfied). Answers are supposed to represent a broad, reflective judgment of people's lives at a particular point in time. Subjective well-being approach analyzes this type of information using more or less complex techniques assuming that (page 34 
Table 9. Spearman's rank correlation coefficients. Poverty measures and quality of life indices.

\begin{tabular}{lcccccc}
\hline & $F G T_{1}$ & $F G T_{2}$ & $F G T_{10}$ & $P_{1}$ & $P_{2}$ & $P_{10}$ \\
\hline$C M Q_{\text {or }}$ & 0.6520 & 0.6299 & $0.2868^{*}$ & 0.6544 & 0.6446 & 0.6495 \\
$C M Q_{-1}$ & 0.6471 & 0.6225 & $0.2990^{*}$ & 0.6495 & 0.6397 & 0.6544 \\
$C M Q_{-2}$ & 0.6397 & 0.5980 & $0.2745^{*}$ & 0.6422 & 0.9902 & 0.6593 \\
$C M Q_{-10}$ & 0.6029 & 0.5319 & $0.1544^{*}$ & $0.6103^{*}$ & 0.5931 & 0.6029 \\
\hline
\end{tabular}

* Not significant at 0.05 level of significance

of Diener ${ }^{12}$ ): "Although subjective well-being is not sufficient for the good life, it appears to be increasingly necessary for it".

Here we compare regional rankings on quality of life, based on the synthetic measurements proposed above, with the regional ranking on life satisfaction. The results suggest that there is no correlation among them. None of the Spearman's rank correlation coefficients are significant. Even more, it is not correlated with poverty rankings presented in Section 3. This result is consistent with studies on adaptation of individuals to new situations by changing their expectations or relative subjective well-being which must be evaluated in a social context being affected for the relative situation of the reference group.

\section{Concluding remarks}

The study of Spanish households' equivalent disposable income in 2012 shows that regions do not substantially alter their position in the ranking when the poverty measurement's sensitivity towards disparities among poor incomes changes. The high values of the Spearman's ranks correlation coefficient confirm this point.

A similar conclusion is obtained by using different composite measures of quality of life. Spanish regions tend to maintain similar positions in the ranking, regardless the ordinal or cardinal approaches to quality of life measurement. The same is true when the possibility of trade-offs between progress and setbacks in different social indicators is modified. Regions on the top and regions on the bottom of the quality of life ranking tend to be the same.

Comparing both kinds of rankings, we get a curious conclusion. Monetary poverty rankings, based on a one-dimensional view (equivalent disposable income), are surprisingly similar to rankings on quality of life, based on a multi-dimensional view (combinations of ten social indicators). It configures poverty measurements as an acceptable proxy for composite measures on quality of life, a possibility that would be desirable to explore in other contexts.

Following the subjective approach to quality of life, we explore the relationships between regional rankings on satisfaction with life and on quality of life, and also on satisfaction with life and on monetary poverty. In this case, none of the Spearman's 
ranks correlation coefficients is significant, in accordance with the literature on this field.

Further research may incorporate other social indicators' weighting schemes or modifications on the selected set of social indicators in order to confirm or refuse the obtained conclusions.

\section{Acknowledgments}

J.L García-Lapresta acknowledges the funding support of the Spanish Ministerio de Economía y Competitividad (project ECO2012-32178) and Consejería de Educación de la Junta de Castilla y León (project VA066U13).

\section{References}

1. A.B. Atkinson, On the measurement of poverty, Econometrica 55 (1987) 749-764.

2. A.B. Atkinson, Poverty in Europe (Blackwell, Oxford, 1998).

3. O. Aristondo, J.L. García-Lapresta, C. Lasso de la Vega, R.A. Marques Pereira, The Gini index, the dual decomposition of aggregation functions, and the consistent measurement of inequality, International Journal of Intelligent Systems 27 (2012) 132152.

4. O. Aristondo, J.L. García-Lapresta, C. Lasso de la Vega and R.A. Marques Pereira, Classical inequality indices, welfare and illfare functions, and the dual decomposition, Fuzzy Sets and Systems 228 (2013) 114-136.

5. G. Beliakov, H. Bustince Sola and T. Calvo Sánchez, A Practical Guide to Averaging Functions (Springer, Heidelberg, 2016).

6. G. Beliakov, A. Pradera and T. Calvo, Aggregation Functions: A Guide for Practitioners (Springer, Heidelberg, 2007).

7. F. Booysen, An overview and evaluation of composite indices of development, Social Indicators Research 59 (2002) 115-151.

8. B. Buhmann, L. Rainwater, G. Schmaus and T.M. Smeeding, Equivalence scales, well-being, inequality, and poverty: sensitivity estimates across ten countries using the Luxembourg Income Study (LIS) database, The Review of Income and Wealth $\mathbf{3 4}$ (1988) 115-142.

9. S.R. Chakravarty and P. Muliere, Welfare indicators: A review and new perspectives. 2. Measurement of poverty, Metron - International Journal of Statistics 62 (2004) $247-281$.

10. W.D. Cook and L.M. Seiford, On the Borda-Kendall consensus method for priority ranking problems, Management Science 28 (1982) 621-637.

11. P. Dasgupta and M. Weale, On measuring quality of life, World Development 20 (1992) 119-131.

12. E. Diener, Subjective well-being: the science of happiness and a proposal for a national index, American Psychologist 55 (2000) 34-43.

13. E. Diener, Guidelines for national indicators of subjective well-being and ill-being, Journal of Happiness Studies 7 (2006) 397-404.

14. D. Donaldson and J.A. Weymark, Propeties of fixed-population poverty indices, International Economic Review 27 (1986) 667-688.

15. Eurostat, Quality of life indicators. Material living conditions. Eurostat Statistics Explained (2016). 
16. J. Foster, J. Greer and E. Thorbecke, A class of decomposable poverty measures, Econometrica 52 (1984) 761-766.

17. V.R. Fuchs, Redefining poverty and redistributing income, The Public Interest $\mathbf{8}$ (1967) 88-95.

18. J.L. García-Lapresta, C. Lasso de la Vega, R.A. Marques Pereira and A.M. Urrutia, A class of poverty measures induced by the dual decomposition of aggregation functions, International Journal of Uncertainty, Fuzziness and Knowledge-Based Systems 18 (2010) 493-511.

19. J.L. García-Lapresta and R.A. Marques Pereira, The self-dual core and the anti-selfdual remainder of an aggregation operator, Fuzzy Sets and Systems 159 (2008) 47-62.

20. J.L. García-Lapresta and R.A. Marques Pereira, The dual decomposition of aggregation functions and its application in welfare economics, Fuzzy Sets and Systems $\mathbf{2 8 1}$ (2015) 188-197.

21. M. Grabisch, J.L. Marichal, R. Mesiar and E. Pap, Aggregation Functions (Cambridge University Press, Cambridge, 2009).

22. A. Hagenaars, The Perception of Poverty (North-Holland, Amsterdam, 1986).

23. S. Jenkins and P. Lambert, Three 'I's of poverty curves and poverty dominance: TIPs for poverty analysis, Research on Economic Inequality 8 (1998) 39-56.

24. M. Martínez-Panero, J.L. García-Lapresta and L.C. Meneses, Multidistances and dispersion measures, in Fuzzy Logic and Information Fusion, eds. T. Calvo Sánchez and J. Torrens Sastre (Studies in Fuzziness and Soft Computing, Springer, 2016), pp. 123-134.

25. M. Nardo, M. Saisana, A. Saltelli, S. Tarantola, A. Hoffman and E. Giovannini, Handbook on Constructing Composite Indicators. Methodology and User Guide, (OECD Statistics Working Papers, 2005/3, OECD Publishing, 2005).

26. OECD, Income Distribution and Poverty in Selected OECD Countries, OECD Economic Outlook 62 (1997) 49-59.

27. OECD, OECD Handbook on Constructing Composite Indicators. Methodology and User Guide (OECD Publications, Paris, 2008).

28. OECD, How is Life? Measuring Well-Being (OECD Publications, Paris, 2011).

29. M. Orshansky, Counting the poor: another look at the poverty profile, Social Security Bulletin 28 (1965) 3-29.

30. S. Ringen, The Possibility of Politics: A Study in the Political Economy of Welfare State (Clarendon Press, Oxford, 1989).

31. B.S. Rowntree, Poverty: A Study of Town Life (Macmillan, London, 1901).

32. J. Salzman, Methodological Choices Encountered in the Construction of Composite Indices of Eeconomic and Social Well-being, (Center for the Study of Living Standards, Ottawa, 2003).

33. A.K. Sen, Poverty: An ordinal approach to measurement, Econometrica 44 (1976) 219-231.

34. A.K. Sen, Issues in the measurement of poverty, Scandinavian Journal of Economics 81 (1979) 285-307.

35. A.K. Sen, Poor, relatively speaking, Oxford Economic Papers 35 (1983) 153-169.

36. D.J. Slottje, Measuring the quality of life across countries. The Review of Economics and Statistics 73 (1991) 684-693 Measuring the Quality of Life Across Countries: A Multidimensional Analysis

37. D. J. Slottje, G.W. Scully, J.G. Hirschberg and K.J. Hayes, Measuring the Quality of Life Across Countries: A Multidimensional Analysis (Westview Press, Boulder, 1991).

38. J.H. Smith, Aggregation of preferences with variable electorate, Econometrica 41 (1973), 1027-1041. 
May 17, $2016 \quad$ 13:11 WSPC/INSTRUCTION FILE $\quad$ IJUFKS-SI-MolpeceresLapresta-1

22 M. Molpeceres-Abella, J.L. García-Lapresta

39. J.E. Stiglitz, A. Sen, and J.P. Fitoussi, Report by the Commission on the Measurement of Economiv Performance and Social Progress (Commission on the Measurement of Economic Performance and Social Progress, Paris, 2009).

40. S. Subramanian, Indicators of Inequality and Poverty (WIDER Research Paper 2000/25, United Nations University, 2004).

41. P. Townsend, Poverty as relative deprivation: resources and styles of living, in Poverty, Inequality and Class Structure, ed. D. Wedderburn (Cambridge University Press, Cambridge, 1974).

42. P. Townsend, The development of research on poverty, in Social Security Research: The Definition and Measurement of Poverty (Department of Health and Social Security, HMSO, London, 1979), pp. 15-26.

43. UNDP, Human Development Report. Work for Human Development (UNDP, New York, 2015). 